\title{
Mean free path effects in the shock-implosion problem
}

\author{
M. J. Goldsworthy and D. I. Pullin \\ Graduate Aeronautical Laboratories, California Institute of Technology, Pasadena, California 91125, USA
}

(Received 24 April 2008; accepted 23 December 2008; published online 11 February 2009)

\begin{abstract}
The effects of finite Knudsen number in the problem of a cylindrically imploding shock wave in a monatomic gas are investigated. Numerical solutions of the flow field are obtained with initial conditions in the ranges $1.25 \leq M_{0} \leq 5$ and $0.005 \leq \mathrm{Kn}_{0} \leq 0.1$ using the direct simulation Monte Carlo method. Results show that as $\mathrm{Kn}_{0}$ decreases and $M_{0}$ increases, the maximum implosion temperature scales increasingly well with the similarity exponent predicted in the Guderley solution for an imploding strong shock in the Euler limit. When the radius of curvature is large, the cylindrical shock thickness is found to be almost identical to the thickness of a planar shock for a given shock Mach number. For small radii of curvature, the cylindrical shock was found to be thicker than the corresponding planar shock. (C) 2009 American Institute of Physics.
\end{abstract}

[DOI: $10.1063 / 1.3075952]$

\section{INTRODUCTION}

The problem of converging cylindrical and spherical shock waves is one of great importance to the study of compressible fluid flows. The propagation of shock waves in regions of variable geometry has obvious applications, for instance, to flows in nozzles and diffusers. Yet an understanding of the behavior of converging shock waves may also be applied to more complex flows such as the problem of Mach reflection. ${ }^{1}$ In addition, under certain approximations, the imploding shock wave conforms to a special class of problems which display self-similarity. Such problems possess the property that a flow-field parameter at any instant in time can be represented as a time-independent transformation of the parameter at any prior times; the gasdynamic equations then reduce to a system of ordinary differential equations in the "similarity variables." This condition may be seen to be far more stringent than that which leads to flows with simple dimensional similarity for a suitable scaling of the initial and boundary conditions. The significance of self-similar flows in compressible fluid dynamics is that they lead to relatively simple relations between dimensional parameters, relations which can then also be used to understand the relative importance of nondimensional groups such as the Mach number and Knudsen number.

The implosion of spherical and cylindrical shocks has been investigated by a number of authors beginning with Guderley. ${ }^{2}$ The gas-dynamic flow is driven by a strong shock focusing onto a region whose radius is very small in comparison to the initial radius. As a weak solution of the Euler equations, the Guderley shock implosion ignores all length scales associated with the flow, including the molecular mean free path on which dissipative effects occur. The strong-shock jump conditions are applied by specifying an initial temperature of zero in the region ahead of the shock. The similarity parameter has the form

$$
\xi=\frac{r}{A t^{n}},
$$

where $r$ is the shock radius at time $t, A$ is a dimensional number to be determined, and $n$ is the similarity exponent. Values of the flow variables at all positions and times may be expressed as functions of $\xi$. The value of the exponent $n$ is a property of the equations of motion and not of the conditions that generate the shock (for instance, see Ref. 3). Values of the exponent $n$ for cylindrical and spherical waves for specific values of the gas constant $\gamma$ have been calculated to an increasing large number of decimal places by a number of authors (see, for instance, Ref. 4). For a monatomic gas with $\gamma=5 / 3, \quad n=0.815624901431225 \quad$ (cylinder) and $n$ $=0.688376822922543$ (sphere).

The Guderley solution can be viewed as an inner, asymptotic strong-shock limit of a more general cylindrical or spherical shock implosion in an Euler fluid. For finite strength shock waves, the approximate theory known as geometric shock dynamics (see Ref. 3) is most useful, though a more exact solution describing a generalized, "universal" shock implosion has been obtained by Ponchaut et al. ${ }^{5}$ These authors used various methods including a series expansion solution in which the Guderley solution forms the leadingorder term. This generalized implosion is asymptotic to the Guderley solution (in space and time) as time increases and the shock strength increases.

These flows are weak solutions to the Euler equations of motion; they assume that the fundamental reference length scale over which dissipative effects occur, the mean free path, is zero. If finite values of this reference length scale $\lambda_{1}$ are to be considered, then it is natural to define a nondimensional parameter, the Knudsen number, relating the mean free path in the undisturbed gas ahead of the shock to the initial shock radius $r_{0}$, 


$$
\mathrm{Kn}_{0}=\frac{\lambda_{1}}{r_{0}} .
$$

With the introduction of $\mathrm{Kn}_{0}$, it is necessary thus to define the starting radius of the problem. Hence, we may no longer expect the flow to be self-similar; the flow-field solution for finite values of $\mathrm{Kn}_{0}$ is in general unknown. We should expect though that, as with the introduction of the finite strength shock, the Guderley solution should be approached both as the flow progresses further from the initial condition and as the initial conditions are chosen to more closely resemble those of the Guderley case. However, for any initial $\mathrm{Kn}_{0}$, as the shock focuses toward the origin, at some time $t$ the local Knudsen number $\mathrm{Kn}=\lambda / r(t)$ based on the instantaneous shock location $r(t)$ becomes large. Hence, the solution of the kinetic flow field in the limit $\mathrm{Kn}_{0} \rightarrow 0$ is not equal to the Guderley solution uniformly in time and space. For the imploding shock, viewed as a solution of the kinetic Boltzmann equation, for any finite $\mathrm{Kn}_{0}$ there will always exist a small domain of space and time surrounding shock impact on the axis, where finite Kn effects will be present. The Guderley solution, viewed presently as an outer limit of a kinetic description, gives infinite shock temperature and shock Mach number at the point of implosion. Experiments of imploding shocks have indeed observed radiative emission from this region which is most likely associated with very high temperatures. ${ }^{6,7}$ Although it is reasonable to suppose that kinetic effects act to lower this temperature, the particular influence of initial Knudsen number on the shock-implosion problem is investigated here.

Presently, we consider the implosion of cylindrical shock for initial conditions corresponding to $1.25 \leq M_{0} \leq 5$ and $0.005 \leq \mathrm{Kn}_{0} \leq 0.1$. The gas is monatomic and nonreacting and the flow is computed numerically using the direct simulation Monte Carlo (DSMC) method of Bird. ${ }^{8}$ Results are presented primarily for the peak temperature, and the validity of a scaling parameter for predicting the maximum temperature based on a combination of $M_{0}$ and $\mathrm{Kn}_{0}$ is investigated. Deviations between the behaviors of cylindrical and planar shocks are discussed through consideration of the variation of shock thickness with $M$ for the case $M_{0}=1.5$ and $\mathrm{Kn}_{0}=0.005$.

\section{PROBLEM FORMULATION}

\section{A. Imploding shock}

The imploding shock may be modeled using any number of initial (boundary) conditions. In general we seek conditions which impose the least number of conditions on the subsequent shock motion, hence those which lead to a solution which rapidly approaches the Guderley solution. A spherically symmetric imploding shock wave has previously ${ }^{8}$ been modeled numerically by impulsively moving a spherical driver "piston" from rest at a constant speed. The gas ahead of the piston is accelerated and forms a converging shock wave. By the principles of shock dynamics, the motion of the piston should have minimal influence on that of the shock after its formation. An analogous piston could also be used to drive a cylindrical shock wave. However, an alterna-

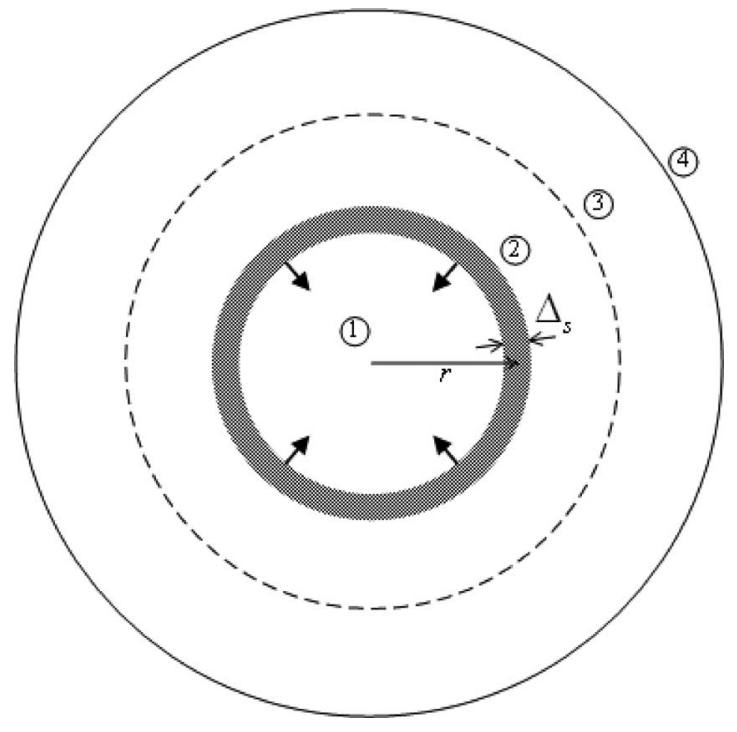

FIG. 1. Schematic of cylindrical Riemann implosion for times $t>t_{0}$. The contact surface (3) and rarefaction (4) travel outwards; the shock wave travels toward the center.

tive method is to consider the shock as originating from a cylindrical Riemann problem. The shock wave is driven by an initial pressure discontinuity across a theoretical dividing membrane; Hornung et al. ${ }^{9}$ showed that multiple combinations of the pressure and speed of sound ratio can be specified, leading to a given initial Mach number $M_{0}$. In the simulations reported here, a pure temperature discontinuity is used.

The cylindrical Riemann problem is shown schematically in Fig. 1. Using the notation commonly associated with the planar Riemann problem, we label the initial states on either side of the discontinuity as states "1" and "4." State " 2 " then corresponds to the conditions behind the moving shock wave and ahead of the contact discontinuity which is labeled state " 3 ." The radius of the shock wave is $r(t)$ and the starting discontinuity is located at a radius $r_{0}$. The shock thickness $\left[\right.$ see Eq. (11)] is represented by the symbol $\Delta_{s}$. The initial condition thus consists of a region $0 \leq r<r_{0}$ with a gas in state 1 and a region $r_{0}<r<\infty$ with a gas in state 4 .

\section{B. Scaling laws in the continuum limit}

It is useful to consider the relation between the instantaneous shock Mach number $M$ and the local Knudsen number Kn using the similarity parameter. From Eq. (1), the shock radius as a function of the time elapsed from some initial radius $r_{0}$ and time $t_{0}$ is given by

$$
\frac{r(t)}{r_{0}}=\left(\frac{t}{t_{0}}\right)^{n} .
$$

$M$ is defined as the speed of the shock relative to the sound speed in the gas ahead of the shock. Likewise, $\mathrm{Kn}$ is defined as the instantaneous radius of the shock relative to the (nominal) mean free path in the gas ahead of the shock, $\lambda_{1}$ $=2 \mu_{1} / \rho_{1} c_{1}$. Here $\mu_{1}$ and $\rho_{1}$ are the gas viscosity and density and $c_{1}$ is the mean thermal speed given by $c_{1}=\sqrt{8 R_{1} T_{1} / \pi}$ where $R_{1}$ is the gas constant. Hence, we have 


$$
\mathrm{Kn}(t)=\frac{\lambda_{1}}{r(t)}=\mathrm{Kn}_{0}\left(\frac{t_{0}}{t}\right)^{n} .
$$

The shock velocity may be used to form an expression for $M$,

$$
M(t)=\frac{\dot{r}(t)}{c_{1}}=\frac{n r_{0}}{t_{0} c_{1}}\left(\frac{t}{t_{0}}\right)^{n-1} .
$$

Combining Eqs. (4) and (5), we obtain the following relation between $\mathrm{Kn}$ and $M$ :

$$
M(t) \mathrm{Kn}(t)^{-(1-n) / n}=M_{0} \mathrm{Kn}_{0}^{(n-1) / n} \equiv C,
$$

where $C$ is a constant for the given initial condition and $M_{0}$ is the Mach number corresponding to the shock which arises from a planar Riemann problem with a specified initial temperature ratio $T_{4} / T_{1}$ across the discontinuity and where the reference sound speed is that of the lower temperature gas.

Equation (6) suggests that if the solutions exhibit similarity when scaled in terms of a parameter $C$, then the corresponding flow-field solutions are increasingly well approximated by the Guderley solution. It may be noted that for the case of $n=0.5$, the parameter $C$ is related to a flow-field Reynolds number $\operatorname{Re}_{0}$. (For the cylindrical implosion in a monatomic gas, $n \approx 0.816$.)

\section{Direct simulation Monte Carlo}

Unsteady numerical solutions of the Boltzmann equation are obtained using the DSMC method. The flow is modeled by computing the motion of a large number of simulator particles. These particles are moved in free flight over a computational time step and collisional energy redistribution is computed statistically at the end of this time step. Kinetic energy exchange in binary collisions occurs without regard to the position of the two participating particles provided that they are separated by no more than a few mean free paths (i.e., they are within the same computational cell). Macroscopic parameters are obtained by sampling simulator particle positions and energies over an appropriately sized region.

The cylindrical Riemann implosion problem as defined above is constructed on the infinite domain $0 \leq r<\infty$. For computational purposes it is necessary to employ an outer boundary of finite extent. The computational domain must be made sufficiently large such that disturbances resulting from the interaction of the rarefaction produced by the Riemannbased initial condition and the outer computational boundary do not reflect and reach the shock either before or during the shock-axis impact.

Flows with cylindrical and spherical symmetries present considerable computational challenges in DSMC, particularly with regards to obtaining accurate information close to the origin where few particles are likely to be present. The number of real particles represented by each simulator particle is often adjusted in proportion to radial location by using variable weighting factors, although this can lead to undesirable numerical effects close to the origin. In the present simulations we do not use variable weighting factors. Instead, the simulator particles are moved in two dimensions over a circular domain and cylindrical symmetry is imposed by computing collisions in a transformed $r-\theta$ space. This means that the flow (and cell structure) is essentially quasione-dimensional, thus enabling a greater resolution of the flow to be obtained for a given computational expense. The trade-off is that the method cannot be used to investigate phenomena associated with shock asymmetries such as the problem of shock stability. ${ }^{10}$

The implosion is modeled using hard-sphere (HS) gas particles so that the mean free path is inversely proportional to the density. The computational cell size and decoupling time step are scaled such that in the region of maximum density and temperature (which occur just after the point of implosion) they are smaller than the local mean free path and mean collision time, respectively. Initially, $10^{7}$ simulator particles are employed in each simulation. The initial condition consists of a temperature discontinuity whose radial location $r_{0}$ is varied to obtain different values of $\mathrm{Kn}_{0}$. Solutions are obtained corresponding to specific values of $\mathrm{Kn}_{0}$ and $M_{0}$. Results are presented in terms of $r_{0}$, the implosion time $t_{0}$ predicted from shock dynamics for the given $M_{0}$ (see the Appendix), and the reference conditions in the initial, undisturbed gas (state 1). All output results are obtained by employing an ensemble averaging procedure using information from multiple, independent DSMC simulations.

\section{Free-molecular solution}

Analytic solutions of the implosion problem for varying values of $M_{0}$ may be obtained in the free-molecular limit $\mathrm{Kn}(t) \rightarrow \infty \forall t$ by considering the simple advection equation of the molecular velocity distribution function $f(\mathbf{c}, \mathbf{r}, t)$ subject to the initial conditions described above,

$\frac{\partial f}{\partial t}+\mathbf{c} \frac{\partial f}{\partial \mathbf{r}}=0 \quad$ with $\begin{cases}f(\mathbf{c}, r, 0)=f_{1}^{*}, & 0 \leq r \leq r_{0}, \\ f(\mathbf{c}, r, 0)=f_{4}^{*}, & r_{0} \leq r<\infty,\end{cases}$

where the "*" denotes the equilibrium Maxwell distribution for the given state. The distribution $f$ is given by the superposition of the two separate and noninteracting streams with distributions $f_{1}^{*}$ and $f_{4}^{*}$. Hence, the low temperature gas may be modeled as the outward free-molecular diffusion of a cylindrical gas cloud of finite extent and the high temperature gas as the diffusion of a gas cloud which extends from the initial radius $r_{0}$ to infinity. The overall density field may be constructed by summing these two contributions corresponding to $i=1,4$ evaluated from the expression

$$
\begin{aligned}
\frac{\rho_{i}(r, \theta, t)}{\rho_{0}}= & \frac{1}{\sqrt{\pi}} \frac{\beta_{i}^{3}}{t^{3}} \int_{-\infty}^{\infty} \int_{0}^{2 \pi} \int_{n_{i}}^{m_{i}} r^{\prime} \exp \left(-\frac{\beta_{i}^{2}}{t^{2}}\left[r^{2}+r^{\prime 2}\right.\right. \\
& \left.\left.-2 r r^{\prime} \cos \left(\theta-\theta^{\prime}\right)+\left(z-z^{\prime}\right)^{2}\right]\right) d r^{\prime} d \theta^{\prime} d z^{\prime},
\end{aligned}
$$

where $\rho_{0}=\rho(r, \theta, t=0)$ on $n_{i} \leq r \leq m_{i}, v_{r} t=r-r^{\prime} \cos \left(\theta-\theta^{\prime}\right)$, $v_{\theta} t=r^{\prime} \sin \left(\theta-\theta^{\prime}\right)$, and $v_{z} t=z-z^{\prime}$ with $n_{1}=0, m_{1}=r_{0}$ and $n_{4}=r_{0}, m_{4} \rightarrow \infty$. The quantity $\beta_{i}$ is the inverse most probable thermal speed given by $\beta_{i}=\left(2 R_{i} T_{i}\right)^{-1 / 2}$. Noting that the field is symmetric in $\theta$ and using the integral representation of the 
Bessel function, this can be expressed in the form

$$
\begin{aligned}
\frac{\rho_{i}(r, t)}{\rho_{0}}= & 2 \frac{\beta_{i}^{2}}{t^{2}} \exp \left(-\frac{\beta_{i}^{2} r^{2}}{t^{2}}\right) \\
& \times \int_{n_{i}}^{m_{i}} r^{\prime} \exp \left(-\frac{\beta_{i}^{2} r^{\prime 2}}{t^{2}}\right) I_{0}\left(2 r r^{\prime} \frac{\beta_{i}^{2}}{t^{2}}\right) d r^{\prime} .
\end{aligned}
$$

(See also Ref. 11. Note that $I_{0}$ is the zeroth-order modified Bessel function of the first kind.)

We may define the overall temperature $T$ at any point in the flow as the mean thermal kinetic energy. The corresponding expression for $T(r, t)$ does not simplify readily for the case of cylindrical symmetry. However, the temperature field may be decomposed into radial, tangential, and cylindrical components, $T_{r}, T_{\theta}, T_{z}$. Since there are no flow-field gradients in the $z$ direction, the cylindrical temperature component is given simply by

$$
T_{z}=\frac{1}{\rho_{1}+\rho_{2}}\left(\rho_{1} T_{1}+\rho_{4} T_{4}\right) .
$$

Hence, Eqs. (9) and (10) may be used to check that the numerical DSMC simulations retain the correct solution in the free-molecular limit. That is, that the boundary conditions do not disrupt the solution until well after the implosion process is modeled.

\section{PLANAR SHOCK THICKNESS}

The variation in shock thickness for the case of a planar shock wave as a function of $M_{0}$ and $\mathrm{Kn}_{0}$, a problem of fundamental interest in gas dynamics, has been studied by numerous authors, of whom we mention only the work of Lilley and Macrossan. ${ }^{12}$ Using DSMC simulations of a monatomic gas, they showed that for a range of gas viscosity models and constant initial upstream Knudsen number (based on an arbitrary reference length), a shock thickness defined by

$$
\Delta_{s}=\left(\rho_{2}-\rho_{1}\right)\left|\frac{d r}{d \rho}\right|_{\max }
$$

initially decreases rapidly with increasing $M$, reaches a minimum, and then increases moderately for high Mach numbers. Further, for constant initial downstream Knudsen number, the shock thickness becomes constant in the limit of high Mach number. These findings support a simple interpretation of the factors governing the planar shock thickness in a monatomic gas.

For given upstream conditions, the shock thickness may be considered to be governed by two factors, the distance between "collisions" in the downstream gas and the efficiency of momentum transfer (the so-called persistence of velocities) in collisions between the upstream and downstream gases. For particles of equal mass, the persistence ratio depends very weakly on the relative velocity in collisions $^{13}$ and rapidly increases to a constant value for high relative energy collisions. For the special case of a HS gas, $\lambda_{2}$ is independent of temperature and hence is inversely proportional to the downstream density,

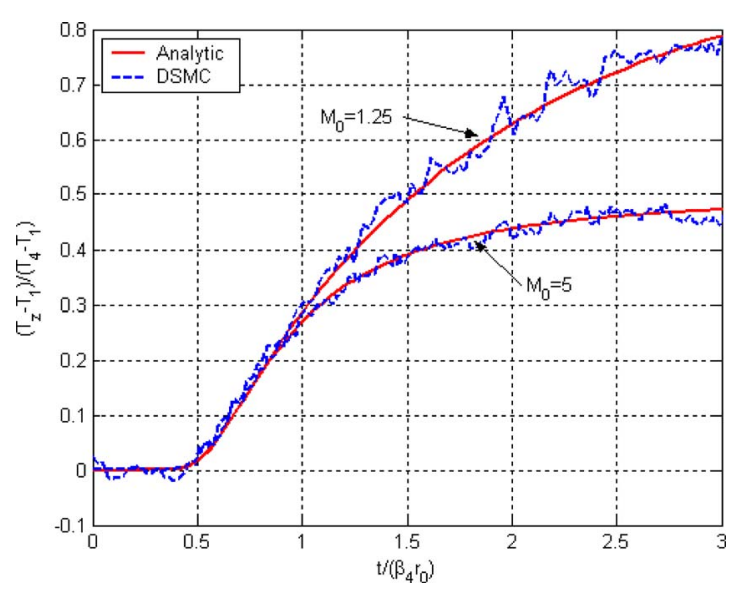

FIG. 2. (Color online) Evolution of normalized free-molecular mean cylindrical thermal energy $\left(T_{z}\right)$ at the origin $(r=0)$ for $M_{0}=1.25$ and $M_{0}=5$; comparison of DSMC simulations (dashed lines) with the analytic expressions from Eqs. (9) and (10) (solid lines). The horizontal axis is the nondimensional time based on the most probable thermal speed of the high temperature gas.

$$
\lambda_{2} \propto \frac{1}{\rho_{2}} \propto \frac{(\gamma-1) M^{2}+2}{(\gamma+1) M^{2}}=f(M) .
$$

Hence, for given upstream conditions, the variation of $\Delta_{s}$ with $M$ will be given largely by $f(M)$, with the actual thickness being slightly larger for small $M$ due to the inefficiency of momentum exchange. In the limiting case $M \rightarrow \infty$, the downstream density is finite and $\Delta_{s} \rightarrow$ const. For real gases whose mean free path is a function of temperature, the shock thickness in the limit of high Mach number thus can be expected to increase slowly with increasing $M$. Although the details of calculating (and hence the appropriate normalization of) $\Delta_{s}(M)$ will depend on the definitions of the mean free path employed, the particular form is necessarily universal.

\section{RESULTS}

\section{A. Free-molecular flow}

For DSMC modeling without radial weighting factors, it is especially desirable to minimize the domain size in the radial direction so as to reduce the total number of simulator particles required. However, disturbances from the imposed boundary condition should not affect the solution at the origin until after the period of interest. The influence of the finite boundary condition is most apparent for the case of free-molecular flow and the time for maximum temperature at the origin (and the implosion time) is, to a first approximation, independent of $\mathrm{Kn}_{0}$.

The cylindrical temperature component $T_{z}$ is a single parameter representing the relative diffusion rates between the two gas streams based on the initial and boundary conditions. Comparisons of the quantity $\left(T_{z}-T_{1}\right) /\left(T_{4}-T_{1}\right)$ at the origin as a function of the nondimensional time $t /\left(\beta_{4} r_{0}\right)$, as calculated using DSMC and the analytic expressions of Eqs. (9) and (10), are shown in Fig. 2 for two simulations with 

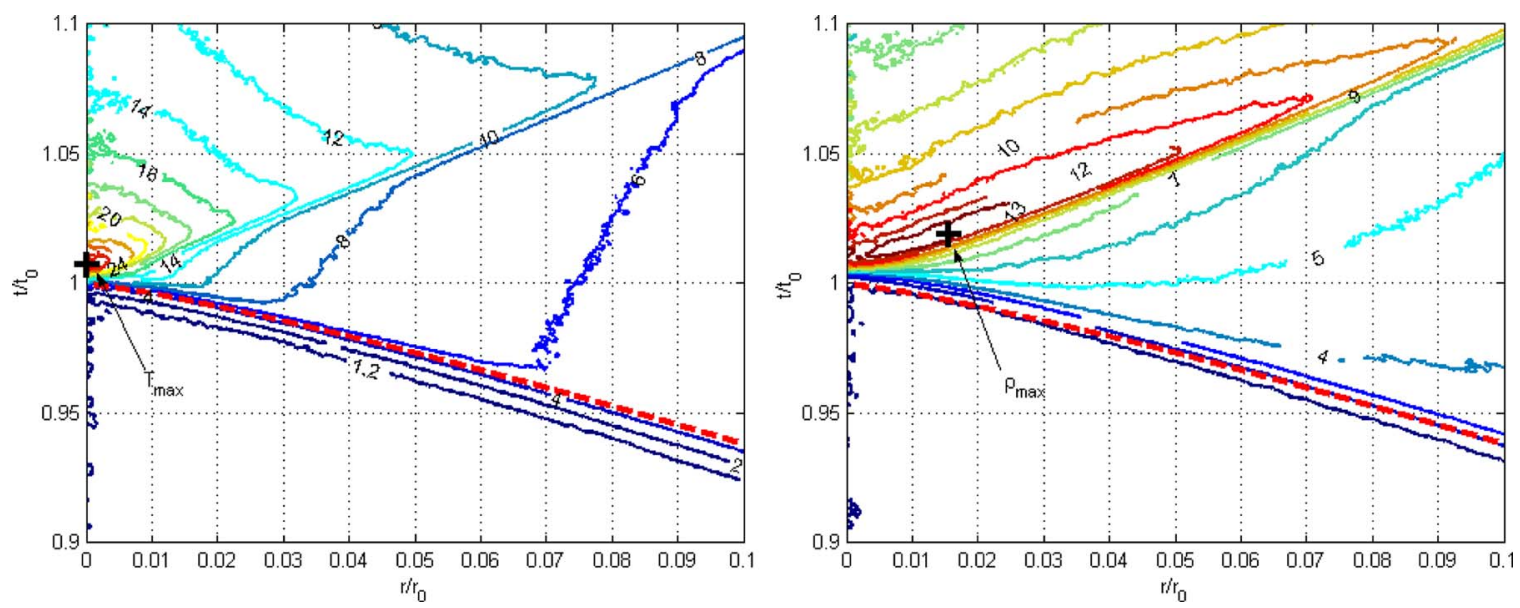

FIG. 3. (Color online) Contours of $T / T_{1}$ (left) and $\rho / \rho_{1}$ (right) in the $r$ - $t$ plane for DSMC simulation case with $M_{0}=2.5$ and $\mathrm{Kn}_{0}=0.005$. The continuum shock location calculated using geometric shock dynamics is overlaid (dashed line). The locations of maximum temperature and density are indicated by the " + " symbol.

$\mathrm{Kn}_{0}=\infty$ and $M_{0}=1.25$ and $M_{0}=5$. The vertical axis is normalized such that in the limit of long times, when $T_{z} \rightarrow T_{4}$, the curves approach a value of 1 . Note that both curves do approach this value; the large difference in the characteristic time scales of the low and high temperature gases for the $M_{0}=5$ case leads to an inflection in the profile at approximately $t /\left(\beta_{4} r_{0}\right) \sim 5$. The maximum temperature for all of the simulations in this study occurred for $t /\left(\beta_{4} r_{0}\right)<1$. It is apparent from the figure that the DSMC results are not influenced by the finite boundary conditions over the time frame of interest.

\section{B. DSMC: Maximum temperature}

Contours of kinetic temperature and density, normalized by the values in state 1 , are shown in the $r$ - $t$ plane in Fig. 3 for the DSMC simulation case with initial conditions of $M_{0}=2.5$ and $\mathrm{Kn}_{0}=0.005$. The time is normalized by the continuum implosion time $t_{0}$ predicted using geometric shock dynamics; the resultant continuum shock trajectory is also overlaid (dashed line). The maximum temperature is located in the cell closest to the origin and occurs at approximately $t=1.005 t_{0}$. The maximum density is located at $r=0.016 r_{0}$ and occurs at $t=1.017 t_{0}$.

Inspection of the simulation results over the range of $\mathrm{Kn}_{0}$ and $M_{0}$ shows that in general $t\left(\rho_{\max }\right)>t\left(T_{\max }\right)>t_{0}$, $r\left(T_{\max }\right)=0$, and $r\left(\rho_{\max }\right)>0$. The ratio $t\left(T_{\max }\right) / t_{0}$ increases as $\mathrm{Kn}_{0}$ increases, although no clear trends are discernible for the variations with $M_{0}$. Given that $t_{0}=t_{0}\left(M_{0}, \mathrm{Kn}_{0}=0\right)$, it is not surprising that no significant deviations occur for changes in $M_{0}$ and further that the DSMC calculated value of $t\left(T_{\text {max }}\right)$ becomes progressively closer to $t=t_{0}$ as $\mathrm{Kn}_{0} \rightarrow 0$. However, from the resolution available it is difficult to identify any consistent trends in the ratios $t\left(\rho_{\max }\right) / t_{0}$ and $r\left(\rho_{\max }\right) / r_{0}$ which appear to display a relatively complex dependence on both $M_{0}$ and $\mathrm{Kn}_{0}$ over the range tested.

The maximum temperature ratio $T_{\max } / T_{1}$ for each DSMC simulation is shown as a function of nondimensional scaling parameter $C$ in Fig. 4. Data points for simulations with the same $M_{0}$ (though different $\mathrm{Kn}_{0}$ ) are shown with the same symbol. It can be seen that as both $\mathrm{Kn}_{0}$ is decreased and $M_{0}$ is increased, $T_{\max }$ increases and $C$ appears to correlate the data with increasing accuracy. The data displayed in Fig. 4 have been fitted to an equation of the form

$$
\frac{T_{\max }}{T_{1}}=A\left[\begin{array}{ll}
M_{0} & \mathrm{Kn}_{0}^{(n-1) / n}
\end{array}\right]^{m} .
$$

The fitting procedure did not assume that the value of exponent $n$ corresponds to the continuum similarity exponent for the problem. Instead, a range of values of $n$ were chosen and linear regression was performed on a log-log transformation of the data to determine the value of the exponent $m$ and the residual of the fit. This procedure was performed successively for data points in the range $C_{\min } \leq C \leq 15$ where five different values for $C_{\min }$ were used. Thus an increasing significance was intentionally given to data points with larger values of $C$. The resultant plot of the residual of the regression versus the exponent $n$ is shown in Fig. 5. It can be seen

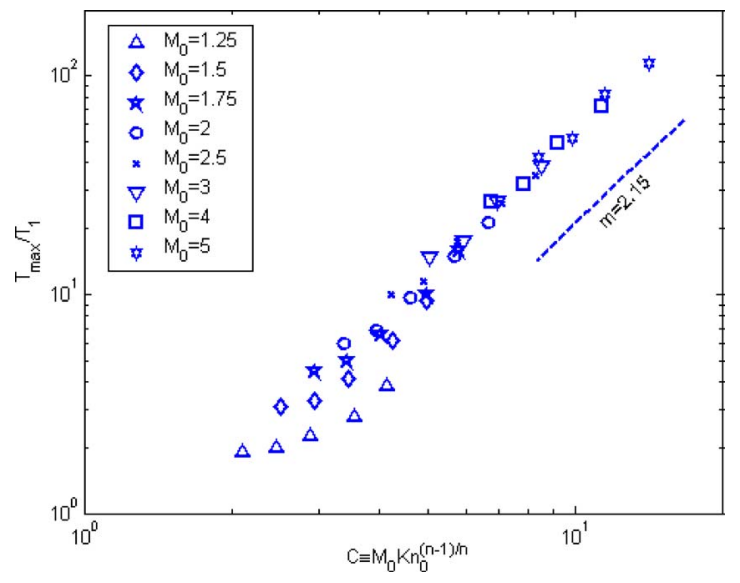

FIG. 4. (Color online) Log-log plot of $T_{\max } / T_{1}$ vs the nondimensional scaling parameter $C$. DSMC results are shown for simulations over the ranges $0.005 \leq \mathrm{Kn}_{0} \leq 0.1$ and $1.25 \leq M_{0} \leq 5 . n=0.8156$. 


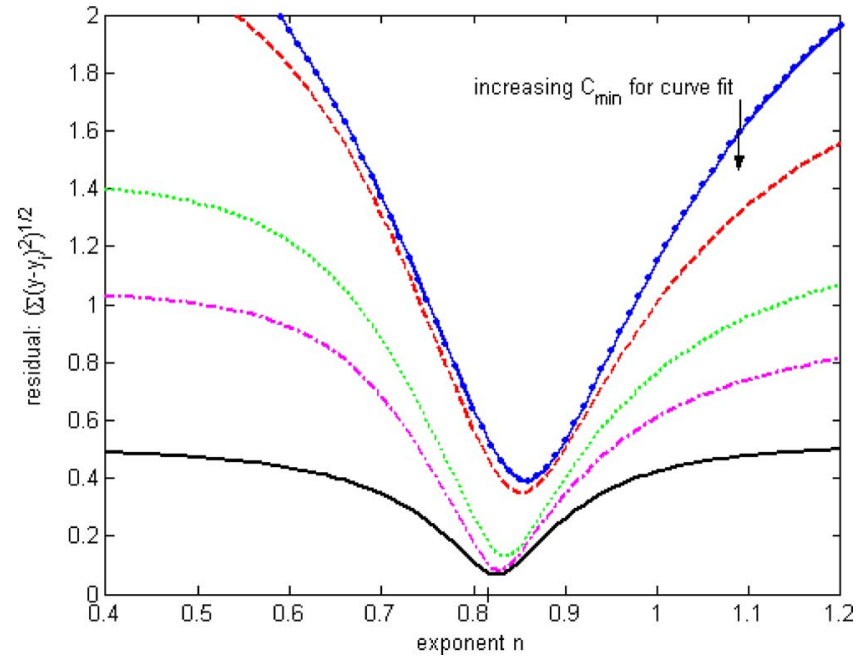

FIG. 5. (Color online) Residual of linear regression fit of $\log \left(T_{\max } / T_{1}\right)$ vs $\log (C)$ as a function of the exponent $n$. Shown are curves for five different data ranges corresponding to $C_{\min } \leq C \leq 15$. The value of $n$ which leads to the best fit for the data points with the largest value of $C_{\min }$ (solid line) is $n=0.82$.

that as the regression is performed over increasing values of $C$, the value of the $n$ which gives the best fit to the data appears to approach the value of the similarity exponent for the case of a cylindrical implosion in a monatomic gas (i.e., $n=0.8156)$. The value of $n$ corresponding to regression over the largest values of $C$ was $n=0.82$ with $m=2.15$ and $A=0.4$.

\section{Shock thickness}

The influence of varying $\mathrm{Kn}_{0}$ on the time variation of the kinetic temperature profile at the origin can be seen in Fig. 6. As $\mathrm{Kn}_{0}$ increases, the arrival time for higher energy "shocked" particles becomes spread over a much larger time frame and $T_{\max }$ is reduced. In addition, the time of maximum

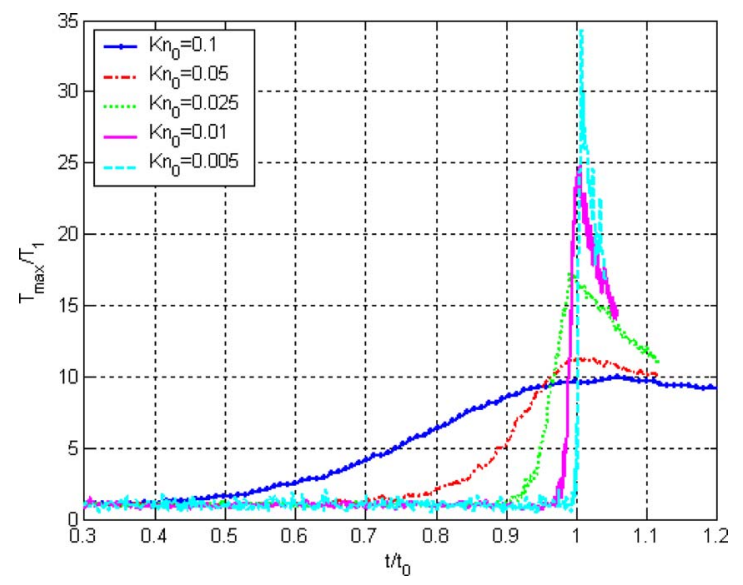

FIG. 6. (Color online) Time history of the normalized kinetic temperature profile in the cell closest to the origin for DSMC simulation case with $M_{0}=2.5$ and varying $\mathrm{Kn}_{0}$.

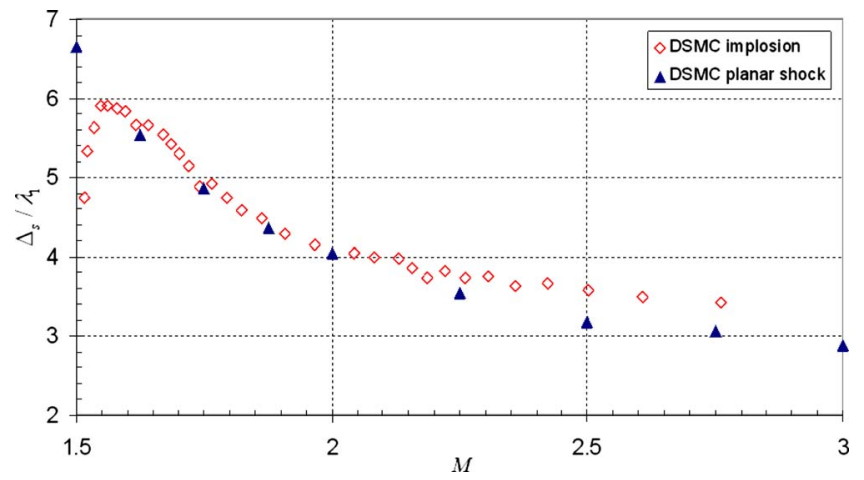

FIG. 7. (Color online) Comparison of normalized shock thickness in a HS gas vs instantaneous shock Mach number for the imploding shock with $M_{0}=1.5$ and $\mathrm{Kn}_{0}=0.005$ and a planar shock calculation.

temperature becomes progressively closer to that predicted using geometric shock dynamics.

It is apparent from these and the previous results that the combination of $M_{0}$ and $\mathrm{Kn}_{0}$ governs the dynamics of the shock implosion and hence the maximum temperature obtained at the implosion point. When $\mathrm{Kn}_{0}$ is large, the collision rate is comparatively less and particles diffuse over much larger distances. Kinetic energy reaching the origin is spread over a larger time window rather than being concentrated at an instant. The effect is more pronounced when the diffusion speed (or we may think in terms of the sound speed) is large in comparison to the bulk gas speed (i.e., when $M_{0}$ is small). Hence, we may expect that the thickness of the shock wave is important in determining the peak implosion temperature.

For the case of converging shock waves, the influence of an instantaneous, finite radius of curvature $r$ on the shock thickness is of interest. We should expect that for $r>>\lambda_{1}, \lambda_{2}$, the planar shock results should be obtained. The variation of $\Delta_{s} / \lambda_{1}$ versus the instantaneous shock Mach number $M$ is shown in Fig. 7 for the case $M_{0}=1.5$ and $\mathrm{Kn}_{0}=0.005$. Also shown are results from separate DSMC simulations of a stationary plane shock wave in a HS gas performed. (Note that in these simulations, $\Delta_{S} \rightarrow \sim 2.25 \lambda_{1}$ and $M_{0} \rightarrow \infty$.)

Given the uncertainty associated with calculating gradients of macroscopic quantities in unsteady DSMC simulations, the comparison shows that for $\sim 1.55<M<\sim 2.1$, the cylindrical and planar shock thicknesses are in fairly close agreement. (Note that for $M=2.1$ and $\mathrm{Kn}=0.05, r \sim 5 \Delta_{s}$.) At $M=3, r \sim \Delta_{s}$; the shock begins to impinge on the origin and the thickness is no longer defined. For $M<\sim 1.55$, the shock structure is still dependent on the initial condition. For $M>\sim 2.1$ the shock structure appears to be influenced by the finite radius of curvature displaying an increased thickness over the planar case. This increased thickness is somewhat counterintuitive; the implosion effectively imposes a geometric restriction on the shock which may be expected to force the shock to become thinner. In reality, collisions among particles with high relative tangential velocities delay the rate of conversion of mean radial velocity to thermal velocity. 


\section{DISCUSSION}

Numerical simulations using the DSMC method employ the dilute gas model in which it is assumed that molecules travel comparatively large distances between collisions relative to a molecular length scale. This condition corresponds to number densities less than $\sim 10^{25} \mathrm{~m}^{-3}$. Hence, notwithstanding computational limitations, we cannot obtain solutions at arbitrarily small values of Kn (i.e., those in the limit $r \rightarrow 0$ ). However, because the maximum density behind the reflected shock is finite, we could conceivably obtain a solution for $\mathrm{Kn}_{0} \rightarrow 0$ by considering the limit $r_{0} \rightarrow \infty$. This is an important distinction. By extension, the maximum temperature "at" the origin is exactly equivalent to the maximum temperature in a region smaller than the local mean free path at the origin provided that the local number density is less than the approximate value given above.

The effects of finite $\mathrm{Kn}_{0}$ on the implosion problem are realized in terms of the initial shock motion due to the applied boundary condition and the motion close to the origin under the influence of finite mean free path. Of these two considerations, the latter is of greater interest since we expect this behavior to be universal in the sense that it is independent of the boundary conditions. Over the limited range of $M_{0}-\mathrm{Kn}_{0}$ space that we have considered here it appears that a form of scaling based on the parameter [Eq. (6)] does indeed apply, at least for the calculation of the maximum temperature. Unfortunately computational constraints mean that simulations with smaller values of $C^{-1}$ were not practical with the computational method employed here.

For an initial value of $C$ corresponding to $M_{0}=5$ and $\mathrm{Kn}_{0}=0.01$, the implosion temperature as predicted from Eq. (13) is approximately 100 times the initial temperature. For a gas initially at room temperature, radiant emission from a reservoir at the corresponding implosion temperature would be significant for many species of interest. In reality, however, electronic excitation, ionization, and radiation would act to lower this peak temperature. Prediction of the actual level of ionization and radiative emission is likely to be sensitive to the initial condition; estimates of the observable spectrum are also dependent on the state of the shock processed gas through which the emission propagates.

The simulations have assumed HS interactions between molecules; such a model is known to result in a limiting planar shock thickness in the case of high Mach number; for more realistic potentials, the shock thickness continues to increase at high Mach number. ${ }^{12}$ Yet the variation of $M$ with $r$ as predicted by the Guderley solution is approximately matched for simulations with finite values of $M_{0}$ and $\mathrm{Kn}_{0}$ and although this solution predicts $M \rightarrow \infty$ as $r \rightarrow 0$ $\left(M \propto r^{1-1 / n}\right)$, the value of $M$ remains small over the majority of the implosion. Indeed for $M_{0}=1.5, M=3$ at $r=0.01 r_{0}$. Hence, for the simulation conditions employed $\left(\mathrm{Kn}_{0} \geq 0.005\right)$, the shock was sufficiently thick such that it began to impinge on the origin before the effects of limiting shock thickness at high Mach number were apparent. Since the Mach-Knudsen number parameter does not include the particular interaction potential model, it is expected to be generally applicable as a scaling parameter. For lower $\mathrm{Kn}_{0}$ flows, more realistic intermolecular potentials are likely to lower the maximum implosion temperature prediction, though in such cases, the modeling of real gas effects are expected to be more important.

\section{CONCLUSION}

The Guderley solution assumes that the length scale for dissipative processes is identically zero, yet a local Knudsen number $\mathrm{Kn}$ based on the shock radius length scale approaches infinity at the implosion point for any initial condition. Hence, it is not necessarily true that the solution of the dissipative flow field in the limit of large initial radius $\left(\mathrm{Kn}_{0} \rightarrow 0\right)$ should approach the Guderley solution. We have investigated the variation of maximum implosion temperature as a function of $\mathrm{Kn}_{0}$ and $M_{0}$ and found that the solution does indeed display a scaling consistent with the Guderley similarity exponent. The length scale over which mechanical conversion of bulk fluid motion into random, disordered motion occurs was shown to be approximately inversely proportional to the square of the maximum implosion temperature for the case $\gamma=5 / 3$. As expected, larger values of $\mathrm{Kn}_{0}$ result in the energy of the shock wave being spread over a larger time. The thickness of the cylindrical shock wave at large radii was found to be comparable to that of a planar shock wave at the corresponding shock Mach number. For smaller radii of curvature, the cylindrical shock wave was thicker. For a real gas for which electronic excitation and ionization occur, the ratio of the peak implosion temperature to the initial temperature is expected to be lower than the values predicted here.

\section{APPENDIX: SHOCK DYNAMICS}

We have made use of the theory of shock dynamics (see Ref. 3) since it gives results extremely close to the true solution yet is much simpler to implement. The motion of the shock is assumed to be driven primarily by the influence of area changes and the interactions between the shock and the downstream flow are assumed to be small. The result is an expression relating the shock Mach number $M$ to the instantaneous cross-sectional area $A$ according to

$$
\frac{M \varphi(M)}{M^{2}-1} \frac{d M}{d r}=-\frac{1}{A} \frac{d A}{d r},
$$

where

$$
\begin{aligned}
& \varphi(M)=\left(1+\frac{2}{\gamma+1} \frac{1-\chi^{2}}{\chi}\right)\left(1+2 \chi \frac{1}{M^{2}}\right) \text { and } \\
& \chi^{2}=\frac{(\gamma-1) M^{2}+2}{2 \gamma M^{2}-\gamma+1}
\end{aligned}
$$

For spherical and cylindrical geometries, $A \propto r^{\nu-1}$ with $\nu=2$ (cylindrical) and $\nu=3$ (spherical). Combining these relations 
and integrating between two locations $r_{1}$ and $r_{2}$ leads (see Ref. 9) to an expression for $r(M)$,

$$
\begin{aligned}
& r\left(M_{2}\right)=r\left(M_{1}\right) Q\left(M_{1}, M_{2}\right), \text { where } \\
& Q\left(M_{1}, M_{2}\right)=\exp \left[-\frac{1}{\nu-1} \int_{M_{1}}^{M_{2}} \frac{m \varphi(m)}{m^{2}-1} d m\right] .
\end{aligned}
$$

Further manipulation gives an expression for the implosion time $t_{0}$ assuming that at $t=0, r=r_{1}$ and $M=M_{1}$ at state 1 and $r=0$ and $M \rightarrow \infty$ at state 2 ,

$$
\begin{aligned}
& t_{0}=\frac{r_{1}}{c} K(v, \gamma, M), \quad \text { where } \\
& K(v, \gamma, M)=-\frac{1}{\nu-1} \int_{M}^{\infty} \frac{\varphi(p)}{p^{2}-1} Q(M, p) d p .
\end{aligned}
$$

In the limit of a strong shock in cylindrical geometry, Eq. (A1) gives $M \propto r^{-\lambda}$. For $\gamma=5 / 3$ we have $\lambda=0.2254$. In the Guderley solution the shock velocity $U$ is proportional to $U \propto r^{(n-1) / n}$. Since the sound speed in the undisturbed gas is constant, we have $n_{s d}=0.816$. This value is extremely close to that obtained from the Guderley solution.
${ }^{1}$ H. Hornung and D. Schwendeman, "Oblique shock reflection from an axis of symmetry: Shock dynamics and relation to the Guderley singularity," J. Fluid Mech. 438, 231 (2001).

${ }^{2} \mathrm{G}$. Guderley, "Starke kugelige un zylindrische verdichtungsstobe in der nahe des kugelmittelpunktes bzw der zylinderachse," Luftfahrtforschung 19, 302 (1942).

${ }^{3}$ G. Whitham, Linear and Nonlinear Waves (Wiley, New York, 1974).

${ }^{4} \mathrm{P}$. Hafner, "Strong convergent shock waves near the center of convergence: A power series solution,” SIAM J. Appl. Math. 48, 1244 (1988).

${ }^{5}$ N. Ponchaut, H. Hornung, D. Pullin, and C. Mouton, "On imploding cylindrical and spherical shock waves in a perfect gas," J. Fluid Mech. 560, 103 (2006)

${ }^{6}$ R. Perry and A. Kantrowitz, "The production and stability of converging shock waves," J. Appl. Phys. 22, 878 (1951).

${ }^{7}$ H. Matsuo, K. Ebihara, and Y. Ohya, "Spectroscopic study of cylindrically converging shock waves," J. Appl. Phys. 58, 2487 (1985).

${ }^{8}$ G. Bird, Molecular Gas Dynamics and the Direct Simulation of Gas Flows (Clarendon, Oxford, 1994).

${ }^{9}$ H. Hornung, D. Pullin, and N. Ponchaut, "On the question of universality of imploding shock waves," Acta Mech. 201, 31 (2008).

${ }^{10} \mathrm{~S}$. Hosseini and K. Takayama, "Experimental study of the RichtmyerMeshkov instability induced by cylindrical shock waves," Phys. Fluids 17, 084101 (2005).

${ }^{11}$ P. Molmud, "Expansion of a rarefied gas cloud into a vacuum," Phys. Fluids 3, 362 (1960).

${ }^{12} \mathrm{C}$. Lilley and M. Macrossan, "DSMC calculations of shock structure with various viscosity laws," in Proceedings of the 23rd International Symposium on Rarefied Gas Dynamics (American Institute of Physics, New York, 2003), Vol. 663.

${ }^{13}$ J. Jeans, An Introduction to the Kinetic Theory of Gases (Cambridge University Press, London, 1940). 